\title{
Efficient proliferation and mitosis of glioblastoma cells infected with human cytomegalovirus is mediated by RhoA GTPase
}

\author{
AHMED A. AL-QAHTANI ${ }^{1,2}$, SAUD ALARIFI $^{3}$, SAAD ALKAHTANI $^{3}$, \\ CHRISTOS STOURNARAS ${ }^{4}$ and GEORGE SOURVINOS ${ }^{5}$
}

\author{
${ }^{1}$ Department of Infection and Immunity, Research Centre, King Faisal Specialist Hospital and Research Centre; \\ ${ }^{2}$ Department of Microbiology and Immunology, Alfaisal University, School of Medicine, Riyadh 11211; \\ ${ }^{3}$ Zoology Department, College of Science, King Saud University, Riyadh 11451, Saudi Arabia; \\ ${ }^{4}$ Department of Biochemistry; ${ }^{5}$ Laboratory of Clinical Virology, Medical School, \\ University of Crete, 71003 Heraklion, Greece
}

Received April 3, 2020; Accepted June 22, 2020

DOI: $10.3892 / \mathrm{mmr} .2020 .11434$

\begin{abstract}
Human cytomegalovirus (HCMV) is a prevalent viral pathogen, which can cause severe clinical consequences in neonates, immunocompromised individuals, patients with AIDS, and organ and stem cell transplant recipients. HCMV inhibits the host cell cycle progress while the immediate-early protein 1 (IE1) tethers to condensed chromatin in mitotic cells. The present study investigated the effect of HCMV on the cell cycle in human glioblastoma cells, as well as the role of RhoA GTPase during mitosis in the same context. Live cell microscopy showed that despite the apparent cell cycle arrest at late stages of mitosis in normal fibroblasts, HCMV-infected U373MG cells successfully went through all stages of cell division. HCMV IE1 protein exhibited a remarkably tight association with mitotic chromosomes from early mitosis to late cytokinesis. Depletion of RhoA significantly impaired the proliferation rate of HCMV-infected U373MG cells; consistent with this observation, the number of cells entering mitosis was also decreased. These results demonstrated the differential behavior of HCMV during mitosis in a normal and a cancer background. Furthermore, RhoA may be a critical component for the efficient cell division of HCMV-infected glioblastoma cells, which subsequently ensures the maintenance of viral genomes.
\end{abstract}

\section{Introduction}

Human cytomegalovirus (HCMV) is a widespread opportunistic pathogen which is normally controlled by the immune

Correspondence to: Professor George Sourvinos, Laboratory of Clinical Virology, Medical School, University of Crete, 71003 Heraklion, Greece

E-mail: sourvino@med.uoc.gr

Key words: human cytomegalovirus, cell cycle, mitosis, RhoA GTPase, glioblastoma cells system. Primary infection is usually asymptomatic or may cause mild symptoms in healthy individuals. However, accumulating evidence supports the concept that periodic reactivation of HCMV can cause severe medical conditions in immunocompromised patients such as transplant, cancer or HIV-infected patients. Furthermore, vertical transmission of HCMV during pregnancy is the leading cause of congenital infection resulting in neurologic damage in neonates [reviewed in $(1,2)]$. The exact mechanisms of establishment, latency and reactivation of HCMV, although fundamental for its successful persistence and dissemination, remain poorly understood.

Lytic HCMV infection is temporally regulated and viral gene expression takes place in three distinct phases, namely the immediate-early (IE), early (E) and late (L) phases, with the onset of each one being induced by the proteins expressed in the previous one. The HCMV immediate-early promoter/enhancer (MIEP) regulates the synthesis of the immediate-early protein 1 (IE1) and immediate-early protein 2 (IE2) which are considered to control all subsequent early and late events in HCMV replication, including reactivation from latency, by antagonizing intrinsic and innate immune responses (3). Both IE proteins are abundantly transcribed and expressed as products of alternative splicing and despite their relative homology, they exert distinct functions; IE1 is essential for efficient viral replication particularly at low multiplicities of infection (4) whereas IE2 has a key role during viral replication (5). HCMV, as a successful opportunistic pathogen, takes control of numerous host pathways such as metabolic and signalling pathways, intrinsic and innate immune responses, apoptosis or even cell cycle arrest strategies to prevent mitotic entry in order to ensure its own maintenance and replication (6). HCMV interrupts the regular order of steps in the cell division machinery by affecting key regulators of cell cycle progression in normal fibroblasts in vitro (7-9). HCMV infected cells have been found to proceed to mitosis but this unscheduled mitotic entry is followed by aberrant spindle formation, early separation of sister chromatids and eventually cell death (10). Interestingly, the HCMV IE1 protein has been known to be associated with condensed chromatin during 
mitosis (11-20). The C-terminal (a.a. 476-491) of IE1 protein has been mapped as the responsible domain for this intriguing association $(17,21)$ however, the molecular mechanism as well as the outcome of this interaction between IE1 and chromatin remains to be determined.

Rho GTPases constitute a family of molecular switches that control fundamental cellular processes such as signal transduction pathways, cellular architecture and morphogenesis, migration, innate and adaptive immunity while deregulation of Rho GTPases have been associated with various human diseases and disorders [reviewed in (22)]. Members of the Rho family, including the RhoA, RhoB and RhoC isoforms have been shown to facilitate critical functions for a productive HCMV infection such as deregulation of cytoskeleton at early stages of infection, signal transduction, formation of the viral assembly compartment or secretion of IL-11, both in normal fibroblasts, the gold-standard cells for studying HCMV in vitro (23-27) or even in a cancer background (28). Combining the involvement of RhoA isoform in the cell division procedure (29-31) along with the mitotic defect in HCMV infected cells, we investigated the role of RhoA GTPAse in the context of human glioblastoma cells productively infected with HCMV. Our results demonstrate that HCMV infected glioblastoma cells, despite the cell cycle arrest observed in normal fibroblasts, are capable to successfully complete all phases of mitosis and cytokinesis. Furthermore, experiments depleting RhoA revealed the active role of this Rho isoform in glioblastoma cell proliferation and mitosis during HCMV lytic infection.

\section{Materials and methods}

Cells and viruses. Human Foreskin Fibroblasts (HFF) and the human glioblastoma U373MG (Uppsala) cell line (kindly provided by Professor Sunyoun Kim, Korea) were authenticated by short tandem repeats DNA profiling and were grown in DMEM (Gibco BRL) supplemented with $10 \%$ foetal bovine serum (Gibco BRL), $100 \mathrm{U} / \mathrm{ml}$ penicillin and $100 \mu \mathrm{g} / \mathrm{ml}$ streptomycin under $5 \% \mathrm{CO}_{2}$ in a humidified incubator at $37^{\circ} \mathrm{C}$. All cells were tested periodically for mycoplasma contamination using the Mycoplasma Plus PCR primer set (Stratagene) and found to be negative. The laboratory strain HCMV AD169 strain as well as the the recombinant HCMV CR401 virus expressing the IE1 fused to EGFP (12) were used in this study. The virus stocks were propagated and titrated on HFF cells according to standard protocols (32). For viral infections, the cells were infected with HCMV at the indicated MOI for $2 \mathrm{~h}$ and then the inoculum was removed and replaced by fresh medium. The autofluoresecent construct pHcRed1-H2A was transfected in U373MG cells to express histone H2A fused to HcRed1 (12) using Lipofectamine 3000 according to the manufacturer's instructions. The initial HCMV CR401 infection of U373MG cells was subsequently followed by transfection of the cells with the pHcRed1-H2A construct.

RhoA knocking down. RhoA protein was knocked down using small interfering RNA (siRNA) oligonucleotides against RhoA mRNA. The siRNA sequence for RhoA was CGGAATGAT GAGCACACAATT and the siRNA sequence for the negative control was GAATAGACCCGTGATAGTACA. U373MG cells were transfected with $2 \mu \mathrm{g}$ of siRNA against RhoA mRNA or the control siRNA using Lipofectamine 3000 according to the manufacturer's recommended protocol. Western blot analysis was used in order to determine the efficient knockdown of RhoA. After $48 \mathrm{~h}$, the culture medium was changed to $1 \%$ serum DMEM and the cells were infected with HCMV AD169 wild type virus. All experiments were in triplicates.

Western blot analysis. The protein lysates from cells were separated by $12 \%$ Tris-glycine SDS-PAGE and electrophoretically transferred onto PVDF membrane. The membrane was incubated in blocking solution at room temperature for $1 \mathrm{~h}$ and subsequently with primary antibodies against RhoA and $\beta$-actin overnight at $4^{\circ} \mathrm{C}$. Visualization of proteins was verified by a chemiluminesence detection method, using the Image Lab Software 6.0.1 (Bio-Rad Laboratories, Inc.).

MTT assay. MTT assay was performed to determine the cell proliferation rate of mock and HCMV infected U373MG after the knockdown of RhoA protein. $1 \times 10^{5}$ cells were transfected with the siRNA scrambled or the siRNA for RhoA and afterwards were infected with HCMV AD169 wild type virus (MOI 2 pfu/cell) or mock infected, in order to quantify the proliferation rate at 1,2 and 3 days after the infection. The MTT reagent (3-(4, 5-dimethylthiazolyl-2)-2, 5-diphenyltetrazolium bromide) (Sigma Aldrich) was used and the cells were incubated for $4 \mathrm{~h}$ at $37^{\circ} \mathrm{C}$ followed by the addition of $150 \mu \mathrm{l}$ MTT solvent (dimethyl sulfoxide-DMSO) for $15 \mathrm{~min}$ and finally the measurement of the absorbance at $590 \mathrm{~nm}$ with a reference filter of $620 \mathrm{~nm}$.

Immunofluorescence analysis. For immunofluorescence, $1 \times 10^{5}$ U373MG cells were transfected with the siRNA for RhoA or with the siRNA scrambled on glass coverslips, were infected with the HCMV AD169 wild type virus at MOI 2 pfu/cell and after 1 day cells were fixed with $4 \%$ PFA and permeabilized with $0.1 \%$ TritonX-100, for $10 \mathrm{~min}$. Cells were stained with the rabbit polyclonal antibody RhoA (sc-179, Santa Cruz Biotechnology, Inc.), followed by a secondary Cy5-labeled goat anti-rabbit antibody (Santa Cruz Biotechnology, Inc.). The nuclei were stained with DAPI. The number of mitotic cells were counted in randomly selected microscopy fields from each condition and acquired with an epifluorescent Leica DMIRE2 microscope equipped with a Leica DFC300FX digital camera.

Statistical analysis. All data shown represent independent experiments carried out in triplicate and are presented as the mean \pm standard deviation. Data were analyzed by one-way ANOVA followed by Tukey post hoc test using GraphPad 8.3.1 software (GraphPad Software, Inc.). $\mathrm{P}<0.05$ was considered to indicate a statistically significant difference.

\section{Results and Discussion}

Recruitment of IE1-72K onto metaphase chromatin in live human fibroblasts and glioblastoma cells. Previous studies have shown that HCMV IE1 associates with condensed chromatin of the host cell during mitosis and cells engaged in viral replication enter into unscheduled mitosis (10-20). The recombinant HCMV CR401 virus expressing IE1 fused 
to EGFP serves as an excellent tool for the visualization of the IE1 protein in live infected cells, exhibiting perfect colocalization with histone $\mathrm{H} 2 \mathrm{~A}$ in mitotic cells (12).

The direct interaction between HCMV IE1 and H2A-H2B histones has been documented, precisely mapping the chromatin-tethering domain (CTD) of IE1 and identifying that several negatively charged $\mathrm{H} 2 \mathrm{~A}$ residues (E56, E61, E64, and D90) composing the nucleosomal acidic pocket, but not acidic residues outside the pocket, selectively direct the $\mathrm{H} 2 \mathrm{~A}$ interaction with the IE1 CTD (21).

Given that HCMV arrests cell cycle progression, we attempted to identify the step in the mitotic process that is impeded by timelapse microscopy. Single cells co-expressing CR401 EGFP-IE1 and HcRed1-H2A, presenting identical localization onto condensed chromosomes, were monitored throughout the course of mitosis. In contrast to the normal cultured fibroblasts, in which mitosis lasts approximately for $1 \mathrm{~h}, \mathrm{HCMV}$ infected fibroblasts remained at several mitotic phases for prolonged times. The IE1-EGFP protein evidently became associated with condensing chromosomes altering its initial diffuse nuclear localization to a compacted pattern alike $\mathrm{H} 2 \mathrm{~A}$ in prophase and prometaphase (Fig. 1Aa-d). IE1-EGFP mitotic chromosomes were properly aligned in metaphase (Fig. 1Ae) however, a severe defect was apparent in anaphase, revealing irregular chromosomal migration which results in a significant number of lagging chromosomes (Fig. 1Af-h) and consequently in failure to complete the cell division. Detailed analysis examining a large number of randomly selected HCMV CR401 EGFP-IE1 mitotic cells, determined their distribution in relation to all mitotic phases (Fig. 1B). The vast majority of the mitotic cells were accumulated in prophase and prometaphase followed by cells progressing to metaphase. A minimal number of cells had advanced to an aberrant anaphase whilst telophase cells were completely absent. Therefore, the progression from metaphase to anaphase appears to be the critical transition step, blocking the efficient cell division and rendering the cells as non-productively infected cells.

The abortive infection observed in mitotic normal fibroblasts prompted us to investigate the fate of cell division in HCMV-infected glioblastoma cells. HCMV expression has been detected in a high percentage of human glioblastomas, the most malignant primary brain tumor [reviewed in (33)] while human glioblastoma cell lines are permissive and support HCMV gene expression (34-36). U373MG glioblastoma cells were infected with HCMV CR401 EGFP-IE1 virus while HcRed1-H2A in those cells served as a marker for condensed chromosomes. Live cell imaging showed that glioblastoma infected cells were capable of successfully completing mitosis. As shown in Fig. 2, IE1-EGFP in tight association with $\mathrm{H} 2 \mathrm{~A}$ was readily detectable in mitotic chromosomes, following the strict sequential order of all phases of cell division and cytokinesis, without encountering the defect observed in fibroblasts. The above experiments demonstrate that there is an apparent differentiation regarding mitotic progression, depending on the cell line background, normal or cancerous.

Several viruses have evolved strategies to arrest cycle progress and inhibit entry to mitosis, presumably in favour of their own functions and overall successful replication and propagation. HCMV represents a characteristic example of a virus causing unscheduled cell division entry and distortion of
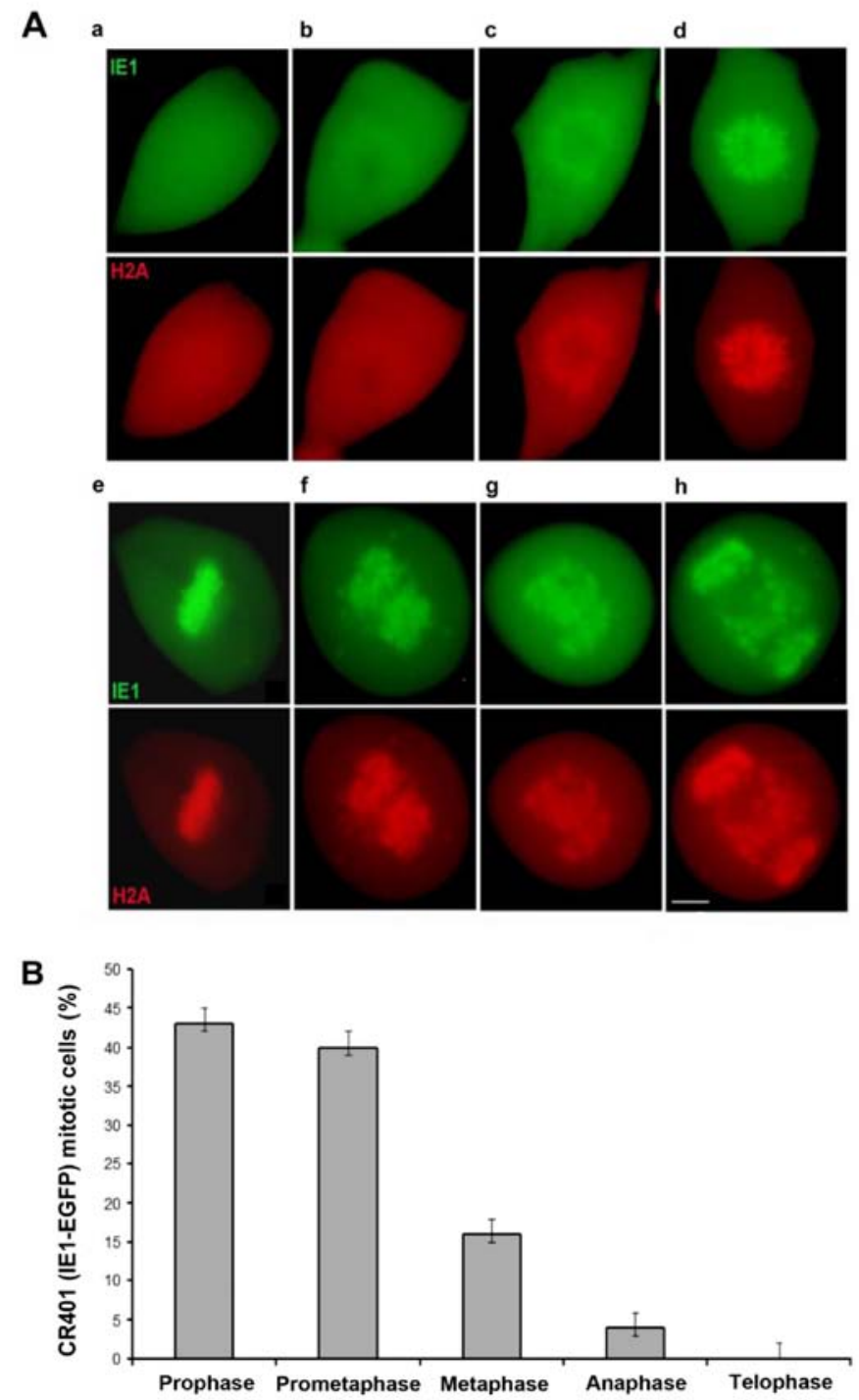

Figure 1. HCMV causes defects in mitotic phases during cell division normal fibroblasts. HFF cells were transfected with pHcRed1-H2A and super-infected with HCMV CR401 (MOI=3 pfu/cell) expressing EGFP-IE1. (A) A single cell co-expressing IE1 (green) and H2A (red) was monitored by timelapse microscopy and still images were obtained every $3 \mathrm{~h}$ showing the defective mitotic progression from (a and b) prophase and (c and d) prometaphase to (e) metaphase and (f-h) defective anaphase, with evident lagging chromosomes. Scale bar, $10 \mu \mathrm{m}$. (B) Distribution of the HCMV-infected mitotic cells at the different phases of cell division. HCMV, human cytomegalovirus; IE1, immediate-early protein 1 .

mitotic structures and subsequent mitotic blockade $(10,12,20)$. Remarkably, the HCMV-induced mitotic restrain observed in normal fibroblasts is entirely abrogated in human glioblastoma cells which unconditionally succeed to divide. In line with our finding, efficient cell division has been observed in an additional glioblastoma cell line permissive to HCMV, T98G cells, which continue to divide, expressing all classes of viral genes. The ability of HCMV to persist within U373MG cells may provide insights into a mechanism for the sustained shedding of the virus. Interestingly, the nuclear antigen 1 of Epstein-Barr virus and the latency-associated nuclear antigen of the Kaposi sarcoma herpes virus (HHV-8), both proteins expressed by gamma herpes viruses, tether metaphase chromosomes, promoting viral genome maintenance during latency and regulation of viral replication $(37,38)$. The specific 


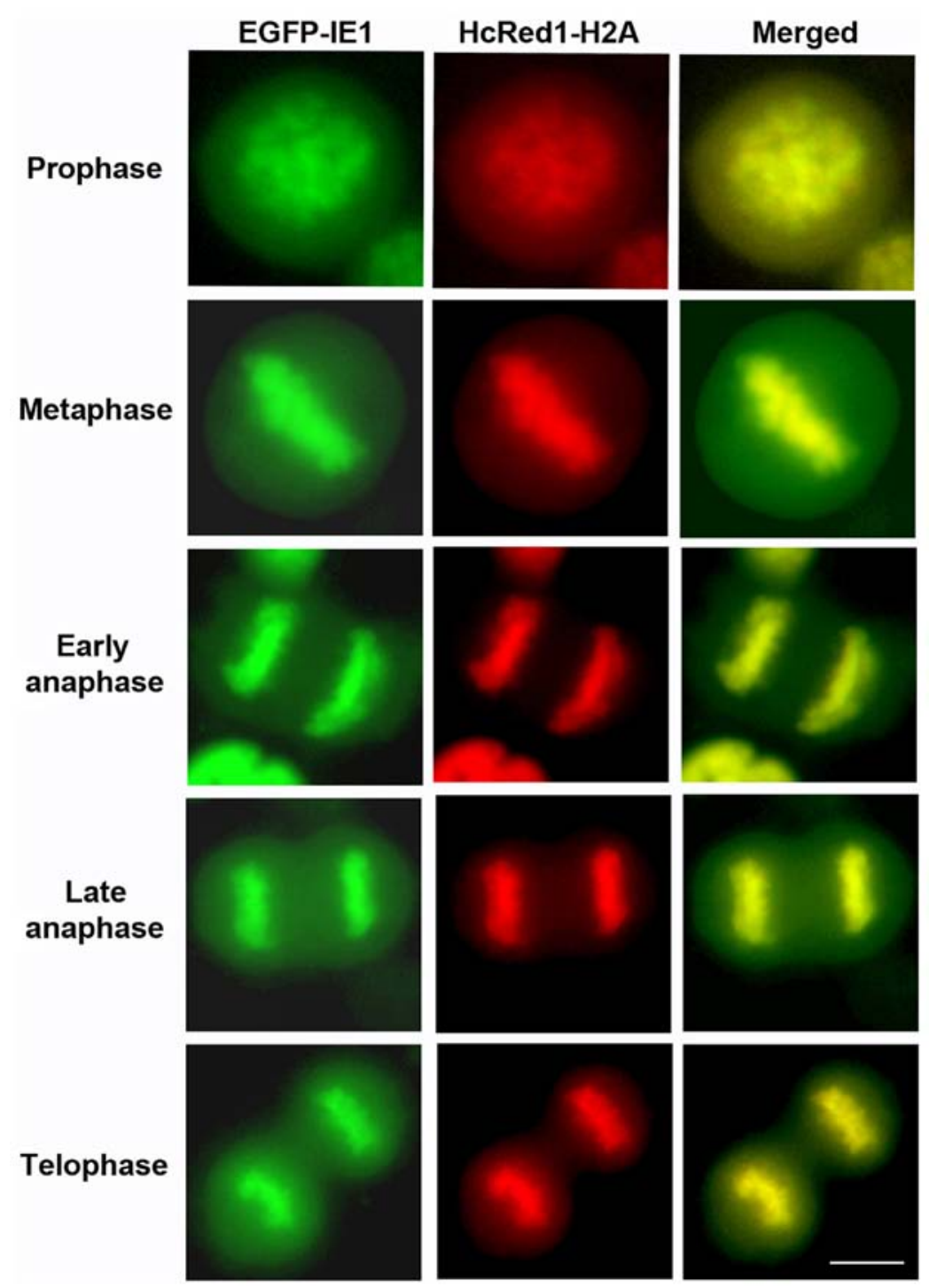

Figure 2. Glioblastoma cells infected with HCMV successfully complete all phases of cell division. U373MG cells were infected with CR401 IE1-EGFP virus (MOI=3 pfu/cell) and transfected with pHcRed1-H2A. Still images were obtained from live cells by timelapse microscopy. Scale bar, $10 \mu \mathrm{m}$. HCMV, human cytomegalovirus; IE1, immediate-early protein 1.

role of IE1 binding onto condensed chromosomes remains to be determined, however it is intriguing to suggest that the chromatin-tethering activity of IE1 facilitates persistence of the viral genomes in dividing cells. Furthermore, the chromosome association properties of IE1 may have an additive role in the uniform segregation of the HCMV genomes into daughter cells.

RhoA GTPase silencing reduces proliferation and mitosis in human glioblastoma cells. RhoA GTPase has been shown to actively be involved in several aspects of HCMV infection (23-28). In addition, RhoA displays crucial roles in several stages of mitosis such as cell cortex stiffening during cell rounding, mitotic spindle formation [reviewed in (39)]. Combining the above, we tested the implication of RhoA in cell division and proliferation of mock or HCMV-infected U373MG cells by knocking down RhoA using the siRNA approach. The successful silencing of RhoA in U373MG cells used as a cell model in the current study, was determined by western blot analysis (Fig. 3A). The proliferation rate of RhoA-depleted cells was significantly decreased for a period of 3 days compared to both parental U373MG and siRNA scramble-treated cells (Fig. 3B). Super-infection of the same set of cells with HCMV AD169 further confirmed the successful replication rate of the RhoA-expressing cells in contrast to the restricted cell division observed in the virally infected RhoA-depleted cells (Fig. 3C). Apart from the growth rate, we were also interested in exploring the mitotic behavior of RhoA-expressing and silenced HCMV infected cells. For that purpose, parental U373MG cells, siRNA scramble and siRNA RhoA cells, were cultured for 2 days and multiple random optical fields were monitored for the detection of mitotic cells. Independently of the siRNA target (siscr or siRhoA), all cells successfully accomplished cell division, with RhoA-depleted cells presenting a significant reduction in the number of mitotic cells compared to the parental and scramble cells (Fig. 3D). The ability of both siRNA scramble and siRNA targeting RhoA to successfully bring about mitosis was also confirmed by immunofluorescence microscopy (Fig. 3E and F). The mitotic rate of the aforementioned siRNA 
A

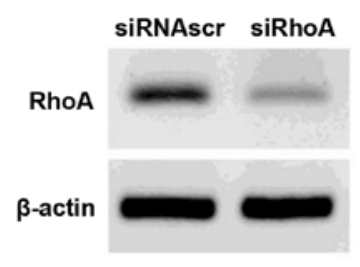

C

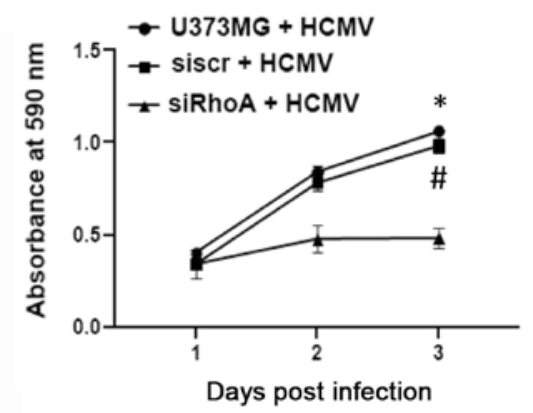

E

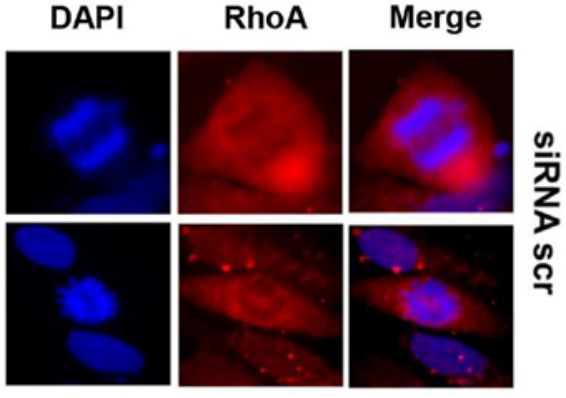

G

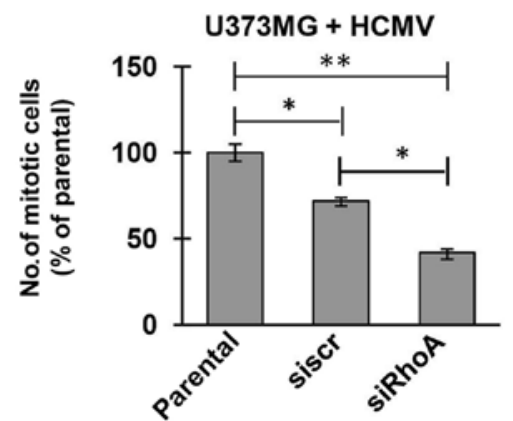

B

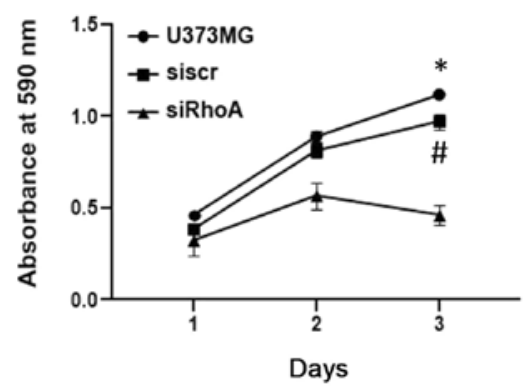

D

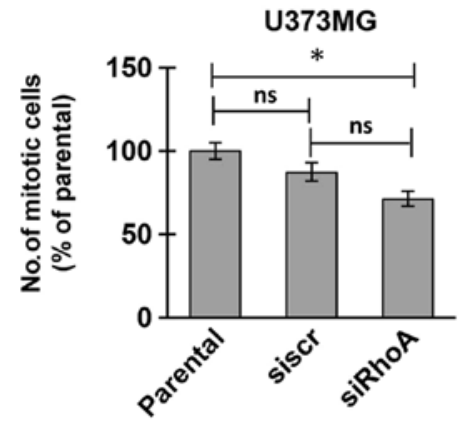

$\mathbf{F}$

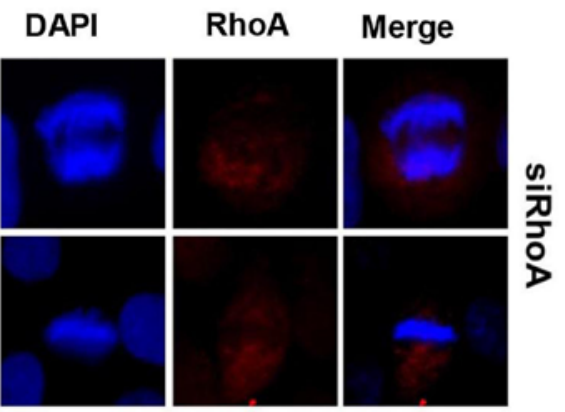

H

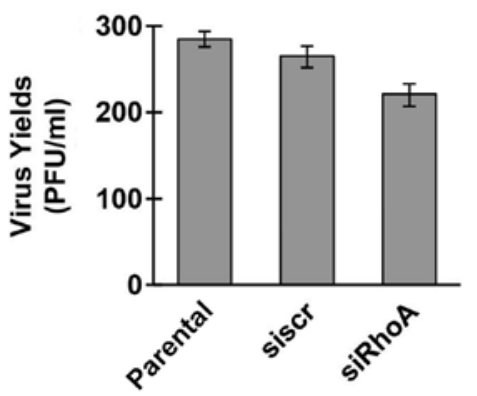

Figure 3. Rho silencing results in defects in cell proliferation rate and mitosis efficiency in glioblastoma HCMV infected cells. (A) Specific knockdown of RhoA in U373 cells was confirmed by western blot from whole protein extracts obtained after transfection with the indicated siRNAs (siRhoA or siRNA scramble) of U373MG cells. Actin served as loading control. (B and C) Relative proliferation rate of parental U373MG, siscr and siRhoA either (B) mock infected or (C) infected with HCMV AD169 (MOI=3 pfu/cell). The proliferation rate of cells was determined by seeding $5 \times 10^{3}$ cells/well and counting the cells in triplicate for up to 3 days by MTT assay. Each experiment was replicated three times and data represent the mean and SE of the three separate experiments. "P<0.05 vs. parental cells; " $\mathrm{P}<0.05$ vs. siscr cells. (D) Proportion of mock-infected mitotic parental U373MG cells, siscr and siRhoA cells compared to each other after counting 10 random microscopy fields. (E) siscr or (F) siRhoA U373MG cells successfully enter mitosis. (G) Proportion of HCMV AD169-infected mitotic parental U373MG cells, siscr and siRhoA cells compared to each other after counting 10 randomly selected microscopy fields. (H) The efficiency of HCMV shedding in siRNA scramble and siRhoA cells was determined by standard plaque assay after titrating the supernatants of the cells on HFF cells. Error bars represent a standard deviation based on results from three experiments. ${ }^{*} \mathrm{P}<0.05 ;{ }^{* * *} \mathrm{P}<0.01$. siscr, siRNA scramble; siRhoA, siRNA RhoA; siRNA, small interfering RNA; DAPI, 4',6-diamidino-2-phenylindole; HCMV, human cytomegalovirus.

silenced cells was further assessed in the context of HCMV infection. In line with the proliferation rates, all groups of infected cells (siscr or siRhoA) proceeded and successfully completed mitosis and cytokinesis. However, the number of mitotic cells with RhoA-depletion was statistically significantly reduced compared to the parental and scramble cells (Fig. 3G). The difference between the HCMV-infected parental and siRNA scramble cells was of less statistical significance, indicating a relative effect of the RNA interference process on the mitotic cells. The aforementioned observation that the siRNA scramble affected mitosis confirmed that although non-targeting controls activate the RNAi machinery allowing a baseline assessment of the effect of the introduction of duplex RNA on gene expression, they can induce a stress response 
within cells. In addition, considering that HCMV is known to trigger multiple cellular stress responses, the difference in number of mitotic cells between the parental and siRNA scramble groups could be due to siRNA/HCMV-induced stress. Moreover, the HCMV shedding was also determined by standard plaque assay, showing an efficient egress of the virus from the glioblastoma cells, presenting no statistically significant difference between siRNA treated and non-treated cells (Fig. 3H). These findings provide a clear evidence for an active role of RhoA GTPase during cell proliferation of uninfected but also remarkably of HCMV infected glioblastoma cells which despite the silencing of RhoA they are still capable of dividing properly. Moreover, cellular DNA synthesis is ongoing, although restrained in RhoA-knockdown cells, even in the presence of the HCMV genomes.

Previous studies have shown an active involvement of RhoA GTPase during several aspects of HCMV cell cycle including cytoskeleton reorganization, viral replication, assembly and egress of the progeny virus (23-28). The current study provides evidence for an additional implication of RhoA in cell proliferation and mitosis of glioblastoma HCMV-infected cells. $\mathrm{RhoA}$ is a critical regulator and ensures the proper formation of mitotic spindle and inhibition of RhoA activity is known to affect distinct phases of the mitotic progression (29-31). We show that glioblastoma cells are cable in overcoming the cell cycle arrest induced by HCMV and our findings on cell proliferation and division after RhoA depletion further expands our knowledge regarding the role of this particular Rho GTPase for viral genome maintenance and shedding.

\section{Acknowledgements}

The authors would like thank Dr Melpomeni Tseliou and Dr Panagiota Dimitropolou (Medical School, University of Crete, Greece) for technical assistance.

\section{Funding}

This project was funded by the National Plan for Science, Technology, and Innovation (MAARIFAH), King Abdulaziz City for Science and Technology, Kingdom of Saudi Arabia (grant no. 12-MED2652-02).

\section{Availability of data and materials}

The datasets used and/or analyzed during the current study are available from the corresponding author on reasonable request.

\section{Authors' contributions}

SaudA and SaadA were involved in methodology, data curation and writing the original draft of the manuscript. AAAQ was involved in methodology, data curation and writing the manuscript. CS and GS were involved in conceptualization of the study, supervised the study, and wrote, reviewed and edited the manuscript. All authors read and approved the final manuscript.

\section{Ethics approval and consent to participate}

Not applicable.

\section{Patient consent for publication}

Not applicable.

\section{Competing interests}

The authors declare that they have no competing interests.

\section{References}

1. Griffiths P: The direct and indirect consequences of cytomegalovirus infection and potential benefits of vaccination. Antiviral Res 176: 104732, 2020.

2. Mocarski ES Jr: Immunomodulation by cytomegaloviruses: Manipulative strategies beyond evasion. Trends Microbiol 10: 332-339, 2002.

3. Adamson CS and Nevels MM: Bright and early: Inhibiting human cytomegalovirus by targeting major immediate-early gene expression or protein function. Viruses 12: 110, 2020.

4. Greaves RF and Mocarski ES: Defective growth correlates with reduced accumulation of a viral DNA replication protein after low-multiplicity infection by a human cytomegalovirus iel mutant. J Virol 72: 366-379, 1998.

5. Marchini A, Liu H and Zhu H: Human cytomegalovirus with IE-2 (UL122) deleted fails to express early lytic genes. J Virol 75: 1870-1878, 2001.

6. Sanchez V and Spector DH: Subversion of cell cycle regulatory pathways. Curr Top Microbiol Immunol 325: 243-262, 2008.

7. Salvant BS, Fortunato EA and Spector DH: Cell cycle dysregulation by human cytomegalovirus: Influence of the cell cycle phase at the time of infection and effects on cyclin transcription. J Virol 72: 3729-3741, 1998.

8. Kalejta RF and Shenk T: Proteasome-dependent, ubiquitin-independent degradation of the $\mathrm{Rb}$ family of tumor suppressors by the human cytomegalovirus pp71 protein. Proc Natl Acad Sci USA 100: 3263-3268, 2003.

9. Song YJ and Stinski MF: Effect of the human cytomegalovirus IE86 protein on expression of E2F-responsive genes: A DNA microarray analysis. Proc Natl Acad Sci USA 99: 2836-2841, 2002.

10. Eifler M, Uecker R, Weisbach H, Bogdanow B, Richter E, König L, Vetter B, Lenac-Rovis T, Jonjic S, Neitzel H, et al: PUL21a-cyclin A2 interaction is required to protect human cytomegalovirus-infected cells from the deleterious consequences of mitotic entry. PLoS Pathog 10: e1004514, 2014.

11. Ahn JH, Brignole EJ III and Hayward GS: Disruption of PML subnuclear domains by the acidic IE1 protein of human cytomegalovirus is mediated through interaction with PML and may modulate a RING finger-dependent cryptic transactivator function of PML. Mol Cell Biol 18: 4899-4913, 1998.

12. Dimitropoulou P, Caswell R, McSharry BP, Greaves RF, Spandidos DA, Wilkinson GW and Sourvinos G: Differential relocation and stability of PML-body components during productive human cytomegalovirus infection: Detailed characterization by live-cell imaging. Eur J Cell Biol 89: 757-768, 2010.

13. Huh YH, Kim YE, Kim ET, Park JJ, Song MJ, Zhu H, Hayward GS and Ahn JH: Binding STAT2 by the acidic domain of human cytomegalovirus IE1 promotes viral growth and is negatively regulated by SUMO. J Virol 82: 10444-10454, 2008.

14. Krauss S, Kaps J, Czech N, Paulus C and Nevels M: Physical requirements and functional consequences of complex formation between the cytomegalovirus IE1 protein and human STAT2. J Virol 83: 12854-12870, 2009.

15. Lafemina RL, Pizzorno MC, Mosca JD and Hayward GS: Expression of the acidic nuclear immediate-early protein (IE1) of human cytomegalovirus in stable cell lines and its preferential association with metaphase chromosomes. Virology 172: 584-600, 1989.

16. Nevels M, Brune W and Shenk T: SUMOylation of the human cytomegalovirus 72-kilodalton IE1 protein facilitates expression of the 86-kilodalton IE2 protein and promotes viral replication. J Virol 78: 7803-7812, 2004.

17. Reinhardt J, Smith GB, Himmelheber CT, Azizkhan-Clifford J and Mocarski ES: The carboxyl-terminal region of human cytomegalovirus IE1491aa contains an acidic domain that plays a regulatory role and a chromatin-tethering domain that is dispensable during viral replication. J Virol 79: 225-233, 2005. 
18. Shin HJ, Kim YE, Kim ET and Ahn JH: The chromatin-tethering domain of human cytomegalovirus immediate-early (IE) 1 mediates associations of IE1, PML and STAT2 with mitotic chromosomes, but is not essential for viral replication. J Gen Virol 93: 716-721, 2012.

19. Wilkinson GW, Kelly C, Sinclair JH and Rickards C: Disruption of PML-associated nuclear bodies mediated by the human cytomegalovirus major immediate early gene product. J Gen Virol 79: 1233-1245, 1998

20. Fang Q, Chen P, Wang M, Fang J, Yang N, Li G and Xu RM: Human cytomegalovirus IE1 protein alters the higher-order chromatin structure by targeting the acidic patch of the nucleosome. Elife 5: e11911, 2016.

21. Mücke K, Paulus C, Bernhardt K, Gerrer K, Schön K, Fink A, Sauer EM, Asbach-Nitzsche A, Harwardt T, Kieninger B, et al: Human cytomegalovirus major immediate early 1 protein targets host chromosomes by docking to the acidic pocket on the nucleosome surface. J Virol 88: 1228-1248, 2014.

22. Hall A: Rho family GTPases. Biochem Soc Trans 40: 1378-1382, 2012.

23. Wang X, Huang DY, Huong SM and Huang ES: Integrin alphavbeta3 is a coreceptor for human cytomegalovirus. Nat Med 11: 515-521, 2005.

24. Seo JY, Yaneva R, Hinson ER and Cresswell P: Human cytomegalovirus directly induces the antiviral protein viperin to enhance infectivity. Science 332: 1093-1097, 2011.

25. Poncet D, Pauleau AL, Szabadkai G, Vozza A, Scholz SR, Le Bras M, Brière JJ, Jalil A, Le Moigne R, Brenner C, et al: Cytopathic effects of the cytomegalovirus-encoded apoptosis inhibitory protein vMIA. J Cell Biol 174: 985-996, 2006.

26. Goulidaki N, Alarifi S, Alkahtani SH,Al-Qahtani A, Spandidos DA, Stournaras C and Sourvinos G: RhoB is a component of the human cytomegalovirus assembly complex and is required for efficient viral production. Cell Cycle 14: 2748-2763, 2015.

27. Alarifi S, Alkahtani S, Al-Qahtani AA, Stournaras C and Sourvinos G: Induction of interleukin-11 mediated by RhoA GTPase during human cytomegalovirus lytic infection. Cell Signal 70: 109599, 2020.

28. Tseliou M, Al-Qahtani A, Alarifi S, Alkahtani SH, Stournaras C and Sourvinos G: The role of RhoA, RhoB and RhoC GTPases in cell morphology, proliferation and migration in human cytomegalovirus (HCMV) infected glioblastoma cells. Cell Physiol Biochem 38: 94-109, 2016.
29. Derksen PWB and van de Ven RAH: Shared mechanisms regulate spatiotemporal RhoA-dependent actomyosin contractility during adhesion and cell division. Small GTPases 11: 113-121, 2020

30. Nakayama Y, Saito Y, Soeda S, Iwamoto E, Ogawa S, Yamagishi N, Kuga T and Yamaguchi N: Genistein induces cytokinesis failure through RhoA delocalization and anaphase chromosome bridging. J Cell Biochem 115: 763-771, 2014.

31. Lian G, Wong T, Lu J, Hu J, Zhang J and Sheen V: Cytoskeletal associated filamin A and RhoA affect neural progenitor specification during mitosis. Cereb Cortex 29: 1280-1290, 2019.

32. Filippakis H, Dimitropoulou P, Eliopoulos AG, Spandidos DA and Sourvinos G: The enhanced host-cell permissiveness of human cytomegalovirus is mediated by the Ras signaling pathway. Biochim Biophys Acta 1813: 1872-1882, 2011.

33. Cobbs CS: Cytomegalovirus and brain tumor: Epidemiology, biology and therapeutic aspects. Curr Opin Oncol 25: 682-688, 2013.

34. Awasthi S, Isler JA and Alwine JC: Analysis of splice variants of the immediate-early 1 region of human cytomegalovirus. J Virol 78: 8191-8200, 2004.

35. Lee K, Jeon K, Kim JM, Kim VN, Choi DH, Kim SU and Kim S: Downregulation of GFAP, TSP-1, and p53 in human glioblastoma cell line, U373MG, by IE1 protein from human cytomegalovirus. Glia 51: 1-12, 2005.

36. Luo MH and Fortunato EA: Long-term infection and shedding of human cytomegalovirus in T98G glioblastoma cells. J Virol 81: 10424-10436, 2007.

37. Marechal V, Dehee A, Chikhi-Brachet R, Piolot T, Coppey-Moisan M and Nicolas JC: Mapping EBNA-1 domains involved in binding to metaphase chromosomes. J Virol 73: 4385-4392, 1999.

38. Piolot T, Tramier M, Coppey M, Nicolas JC and Marechal V: Close but distinct regions of human herpesvirus 8 latency-associated nuclear antigen 1 are responsible for nuclear targeting and binding to human mitotic chromosomes. J Virol 75: 3948-3959, 2001.

39. Chircop M: Rho GTPases as regulators of mitosis and cytokinesis in mammalian cells. Small GTPases 5: e29770, 2014.

This work is licensed under a Creative Commons

Attribution-NonCommercial-NoDerivatives 4.0 International (CC BY-NC-ND 4.0) License. 